\title{
Identification of the risk factors and microbes responsible for inducing central line blood stream infection at a ministry of health hospital in Egypt
}

\begin{abstract}
Aim of the study: To identify the risk factors and microbes responsible for inducing central line blood stream infection and study the antibiotic sensitivity patterns of the isolated organisms in critically ill patients in ICU setting.

Design setting and participants: A descriptive exploratory design was used utilizing non probability convenient sample consisting of 120 adult patients who underwent insertion of central venous catheter in the Intensive Care Units at one of a Ministry of Health Hospital in Egypt between September 2016 and September 2017.

Methods: Patients' demographic data, clinical characteristics, blood culture, antibiotic sensitivity test and catheterrelated data were utilized in data collection from patients, records and microbiology laboratory.

Results: Out of 120 central line catheters sent for culture from patients during and seven days after insertion revealed no growth during insertion and $10.83 \%$ were colonized after insertion. Of the 13 catheter infections cases, it revealed that 10(76.9\%) were Staphylococcus epidermis and 3 (23.07\%) were Staphylococcus aureus. In addition, most infected patients were overweight and obese, diabetic, had sub-clavian route, and their length of hospital stayed more than 7 day.

Conclusions and recommendations: Conclusions and recommendations: Central line venous catheter related factors are the contributing factors for inducing infection of cases with Staphylococcus epidermis in the present study. Moreover, replication of the study and further researches are recommended on a larger probability sample to validate findings
\end{abstract}

Keywords: risk factors; microbes; central line, bloodstream infection (BSI)

\section{Background}

Central venous catheters are commonly utilized in critically ill patients [1] to administer drugs, intravenous fluids, blood transfusion and hemodynamic monitoring [2,3]. Although the benefits of central venous catheters, it may induce many complications such as blood stream infection, bleeding, thrombosis, and mortality [3,4]. Various factors can lead to CVC-associated infections that encompass decreased immunity of patients, site of CVC insertion, the number of lumens, and prolonged hospitalization [5].

Two main pathways that have been proven for causing central venous catheter related blood stream infection including; the extralumenal and intralumenal entrance routes [6] .Organisms that lives on the skin surface can migrate from the site of central venous catheter insertion to the catheter tip causing growth of organisms and making colony; this is the most common route of infection for short term CVCs . Regarding keeping and utilizing central venous catheters at the insertion site for more than 15 days, this may cause migration of organisms from the insertion site to the internal lumen of the catheter [7].

Another cause of infection of central venous lines is a lack of health team embers' knowledge and practices who afford care for patients with central venous catheters $[8,9]$.

Nurses play an important role in providing care of patients in patients connected with central venous catheters. They assist in central line insertion, care of dressing, withdrawing a venous blood sample, and delivery of drugs. As well, Critical care nurses teach the patients and their family about the care of central venous catheters. Therefore, they contribute to the decline of infection [10]. Besides, integrated infection control programs, including supervision of health-related infections (HAIs)
Nahla Shaaban Khalii ${ }^{1 *}$, Manal Sayed Ismaeel ${ }^{2}$ \& Gehan Abd Naby Mohamed $^{3}$

${ }^{1,2}$ Critical care and Emergency Nursing, faculty of Nursing, Cairo University, Egypt.

${ }^{3}$ Al Haram Hospital,Giza, Egypt

*Author for correspondence: nahlakhalil28@yahoo.com 
have brought to a notable decline in the rate of infections in the ICU with resulting lowered health care costs [11].

\section{Aim of the study}

The aim of this study was to identify the risk factors and microbes responsible for inducing central line blood stream infection and study the antibiotic sensitivity patterns of the isolated organisms in critically ill patients in ICU setting.

\section{Material and Methods}

\section{- Design}

A descriptive exploratory design was utilized. Consecutive critically ill patients were engaged into the study between September 2015 and September 2016.

\section{Setting}

The study was conducted at critical care units at a ministry of health hospital in Giza, Egypt. That hospital manages 5000 cases /year. The nurse patient proportion is 1:3.

\section{- Sample}

A convenient sample consisting of 120 patients admitted to the intensive care unit and was subjected to insertion of central venous catheters at one of Ministry of Health Hospitals. The inclusion criteria were as follows: adult patient over 18 years and had no blood stream infection before insertion of central venous catheters. Blood culture were collected after immediate insertion of central venous catheter and seven days after insertion.

\section{- Methods}

The following tools were utilized for data collection

-Demographic and patients' clinical profile: it included data such as age, sex, body mass index, mobility, pre-morbid disease, length of hospital stay.

-central venous catheter related data: it included site of insertion, number of catheter lumen, use of guide wire, duration of insertion , duration of Cather in situ, and location of insertion

-Blood culture Specimen collection: Collected samples were aseptically transferred to the laboratory, cultured in fresh Nutrient Agar Media plates and were incubated within 24 hours of their collection. After incubating for
24 hours at $37^{\circ} \mathrm{C}$, cultures were stored at $2-8^{\circ} \mathrm{C}$ for further examinations.

-Identification test of isolated organisms:

Selective media were used. Such as, MacConkey Agar was used to identify the organisms such as Staphylococcus aureus, Staphylococcus epidermidis and others.

\section{- Antibiotic sensitivity test}

Antibiotic sensitivity test of bacterial isolates on 23 commonly used antibiotics were done on MullerHinton agar medium by disk diffusion technique according to National Committee for Clinical Laboratory Standards (NCCLS) as indicated by 12 utilizing Disc of antibiotics such as Erythromycin $(15 \mu \mathrm{g})$, Cephalexin $(30 \mu \mathrm{g})$, Ceftriaxone $(30 \mu \mathrm{g})$, Chloramphenicol $(30 \mu \mathrm{g}$ Piperacillin $(100 \mu \mathrm{g})$, Colistin $(50 \mu \mathrm{g})$, Amoxicillin $(30 \mu \mathrm{g})$, Penicillin $(10 \mu \mathrm{g})$, Ceftazidime $(30 \mu \mathrm{g})$, Cloxacillin $(1$ $\mu \mathrm{g})$, Nalidixic acid $(30 \mu \mathrm{g})$, Ciprofloxacin $(5 \mu \mathrm{g})$, Cotrimoxazole $(25 \mu \mathrm{g})$ Levofloxacin $(5 \mu \mathrm{g})$, Streptomycin $(25 \mu \mathrm{g})$, Cefotaxime $(30 \mu \mathrm{g})$, Gentamycin $(10 \mu \mathrm{g})$, Amikacin $(30$ $\mu \mathrm{g})$, Neomycin $(30 \mu \mathrm{g})$, Vancomycin (30 $\mu \mathrm{g})$, Azithromycin $(30 \mu \mathrm{g})$, Tetracycline $(30$ $\mu \mathrm{g})$, Imipenem $(10 \mu \mathrm{g})$ and are put on each isolate cultures respectively and incubated at $37^{\circ} \mathrm{C}$. Later, after 24 hours of incubation, the diameter of zone of inhibition that surrounds the antibiotics was calculated according to the instruction of NCCLS.

\section{Content validity}

Content validity of the investigators' developed tools were provided by a panel of ten health care experts including 3 critical care nursing specialists, 4 working critical care nurses and three infection control to evaluate the content validity of the observational checklists. The questionnaire and checklists were reviewed based on their opinions However; we employed the content validity index (CVI) to investigate the quantitative content validity of the established data collection tools. Concerning content validity ratio $(\mathrm{CVR}$,$) we sought the$ experts to score each item on a 3-point Likert scale-Essential (scored 1), Useful but not essential (scored 2) and not essential (scored 3). In respect to the number of experts and the critical values of CVR as proposed by Lawshe (1975). We chose elements having a CVR of 0.5 or more [10]. For calculating content validity 
index in our study, we sought the elected professionals to investigate, clarity, relevancy and simplicity of each element by using a 4-point Likert scale [14]. The critical value point for CVI was accepted at 0.79 [15]. Items having a CVI of 0.79 or higher were included in the final form of questionnaire and observational such as insertion of CVC catheter, dressing care, and IV administration of fluids and medication.

\section{Ethical consideration}

Ethical approval for the research was obtained from the proper authorities. All patients were called for to take part in the study. Informed consent was described and simplified to each patient to receive his/ her agreement as a member. Conscious patients had given written informed approval to take part in the study before insertion of central venous catheters. While semiconscious and comatose patients, signed informed consents were taken from their relatives. Thereafter, during insertion and after removal of the central venous catheter, blood culture specimens were collected from each case to be dealt with for checking bloodstream infection. Anonymity and Confidentiality of patients' data were retained throughout the study.

\section{Data analysis}

Statistical analysis was completed involving the statistical package for social sciences (SPSS) version 21. Continuous data was described as the mean and standard deviation (SD), while the categorical and nominal was declared as frequencies and percentages. Chi-square analysis was utilized for correlates qualitative data and a value of $\mathrm{p}<0.005$ was considered significant.

\section{Results \\ - Demographic and clinical characteristics of the studied subjects}

The demographic and clinical characteristics for the studied patients are showed in TABLES 1 and 2 respectively among the overall population $(n=120)$ and central related blood stream infection (CRBSI) cases ( $n=13)$. In summary, the mean age in years among study population was 52.2 years and that among patients with CRBSI were 60 . The commonest pre-morbidity among the study population and patients with CRBSI was diabetes (31.7\% and $53.8 \%$ respectively). Concerning the condition for central venous line insertion among study population and patients with CRBSI were emergent $(90 \%$ and 92.3\% respectively). Overweight and obese patients were common in both population and CRBSI cases ( $43.3 \%$ versus $46.1 \% \& 25.8 \%$ versus $23.07 \%$ respectively).Dependent patients were seen among all patients and patients with CRBSI (63.3\% versus 69.24\%). Eventually, the hospital length of stay among all patients with

\section{TABLE 1. Clinical characteristics of patients.}

\begin{tabular}{|c|c|c|}
\hline Variables & $\begin{array}{l}\text { Overall Number of patients } \\
\qquad 120(100 \%)\end{array}$ & $\begin{array}{l}\text { Number of Catheter related blood stream infections } \\
\qquad \text { cases } 13(10.8 \%)\end{array}$ \\
\hline \multicolumn{3}{|l|}{ Gender } \\
\hline Male & $69(57.5 \%)$ & $8(61.5 \%)$ \\
\hline Female & $51(42.5 \%)$ & $5(38.5 \%)$ \\
\hline Mean age in years & Mean $=52.2 ; S D=16.5$ & $60 \mathrm{SD}=15.5$ \\
\hline \multicolumn{3}{|c|}{ Duration of hospital stay } \\
\hline$<10$ days & $81(67.5 \%)$ & $0(0 \%)$ \\
\hline$>10$ days & $39(32.5 \%)$ & $13(100 \%)$ \\
\hline Mean hospital stay & 9 days $S \mathrm{D}=6.6$ & 10 days $S D=4.6$ \\
\hline \multicolumn{3}{|l|}{ Pre-morbidities } \\
\hline Diabetes & $38(31.7 \%)$ & $7(53.8 \%)$ \\
\hline Hypertension & $82(68.3 \%)$ & $6(46.2 \%)$ \\
\hline \multicolumn{3}{|l|}{ Reason for Indications } \\
\hline Emergent indication & $108(90 \%)$ & $12(92.3 \%)$ \\
\hline Routine replacement & $12(10 \%)$ & $1(7.7 \%)$ \\
\hline \multicolumn{3}{|l|}{ Body mass index } \\
\hline Under weight & $4(3.3 \%)$ & $0(0 \%)$ \\
\hline Normal weight & $33(27.5 \%)$ & $4(30.7 \%)$ \\
\hline Over weight & $52(43.3 \%)$ & $6(46.1 \%)$ \\
\hline Obese & $31(25.8 \%)$ & $3(23.07 \%)$ \\
\hline
\end{tabular}


CRBSI was more than 10 days when compared to the overall population.

\section{Identification of microbes for the studied patients}

Of the 120 patients enrolled in this study, all subjects' blood culture displayed no growth of microorganisms at the end of the insertion procedure. On the other hand, 13 (10.8\%) developed the growth of bacteria 7 days after insertion of central venous catheters (FIGURE 1). Concerning the finding pertinent to Bacterial separates from blood cultures, it

\section{BLOOD CULTURE 2-AFTER INSERTION OF C.V.C}

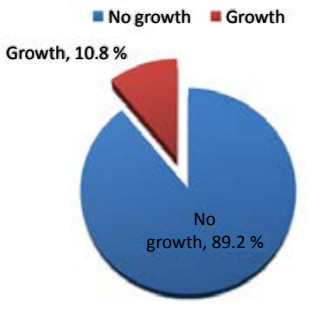

FIGURE 1. Percentage distribution of patients' blood culture 7 days after CVC insertion of $(\mathrm{N}=120)$.

TABLE 2. Bacterial isolates from blood cultures seven days after CVC insertion.

\begin{tabular}{|c|c|c|c|}
\hline \multirow{2}{*}{ Variable } & \multirow{2}{*}{ Values } & \multicolumn{2}{|c|}{$\begin{array}{c}\text { Number of positive } \\
\text { Cultures } N=13\end{array}$} \\
\hline & & NO. & $\%$ \\
\hline \multirow{2}{*}{ pathogen } & $\begin{array}{l}\text { Staphylococcus } \\
\text { Epidermidis }\end{array}$ & 10 & 76.93 \\
\hline & $\begin{array}{c}\text { Staphylococcus } \\
\text { Aureus }\end{array}$ & 3 & 23.07 \\
\hline
\end{tabular}

revealed that $10(76.9 \%)$ were Staphylococcus epidermis and 3 (23.07\%) were Staphylococcus aureus (TABLE 2).

\section{Central venous catheter characteristics and risk factors among studied patients}

Of the 120 patients enrolled in this study, all patients had triple lumen catheter and were inserted in ICU setting. Regarding duration of central venous catheter insertion, all 13 CRBSI cases experienced more than 15 minutes utilizing guide wire during insertion. Moreover, more than half of patients' central venous catheters were inserted via jugular vein (52.5\%). However, CRBSI incidence was higher in patients with the subclavian route $(61.54 \%)$ when compared to jugular (38.46\%). Regarding the duration of the catheter in place among all patients and patients with CRBSI, more than two-thirds of the overall population $(67.5 \%)$ experienced less than 7 days. However, all the CRBSI cases experienced more than seven days as shown in TABLE 3.

Regarding studying the cultural sensitivity of bacterial isolates to antibiotics in the current study, it showed that all isolates were sensitive to Vancomycin, Amikin, and Levofloxacin... Eighty percent of bloodstream infection samples were resistant to Amoxycillin Clavulanic acid, 60\% Meropenem, 50\%, Clindamycin and ceftriaxone, $40 \%$ Gentamicin, Penicillin and Ofloxacine and 30\% Imipenem.

\section{TABLE 3. Catheter related factors for the studied patients.}

\begin{tabular}{|c|c|c|}
\hline Variables & $\begin{array}{l}\text { Overall Number of patients } \\
120(100 \%)\end{array}$ & $\begin{array}{l}\text { Number of Catheter related blood stream } \\
\text { infections cases } 3(10.8 \%)\end{array}$ \\
\hline \multicolumn{3}{|l|}{ Insertion site } \\
\hline Jugular & $63(52.5 \%)$ & $5(38.46 \%)$ \\
\hline Sub-clavian & $57(47.5 \%)$ & $8(61.54 \%)$ \\
\hline \multicolumn{3}{|c|}{ Use of guide wire } \\
\hline Yes & $113(94 \%)$ & $13(100 \%)$ \\
\hline No & $7(6 \%)$ & $0(0 \%)$ \\
\hline \multicolumn{3}{|c|}{ Number of lumens } \\
\hline Single & $0(0 \%)$ & $0(0 \%)$ \\
\hline Triple & $120(100 \% 0$ & $13(100 \%)$ \\
\hline \multicolumn{3}{|c|}{ Duration of catheter in place } \\
\hline$<7$ days & $81(67.5 \%)$ & $0(0 \%)$ \\
\hline$>7$ days & $39(32.5 \%)$ & $13(100 \%)$ \\
\hline \multicolumn{3}{|c|}{ Duration of insertion } \\
\hline$<15$ minutes & $13(10.83 \%)$ & $0(0 \%)$ \\
\hline$>15$ minutes & 107(89.17\%) & $13(100 \%)$ \\
\hline
\end{tabular}




\section{Discussion}

This study analyzed the incidence, clinical and microbiological profiles, and predisposing factors for the development of catheter-related infections in a ministry of health hospital in Egypt. Of the 120 patients enrolled in this study, $13(10.8 \%)$ developed the growth of bacteria after removal of central venous catheters (FIGURE 2). These findings are agreeing with that of 16 who examined healthcare-associated bloodstream infections in critically ill patients and utilized the descriptive cross-sectional database to evaluate concordance with clinical site isolates and found that CLBSI accounted for $6.0 \%$.

As well, our findings are consistent with [6] who reported the blood infection stream rate was 10.2 per 1,000 catheters/day as well [17] who revealed the bloodstream infection rate 1.4 per 1000 catheter-day. Similarly, our findings are in line with previous studies done by [1820] who reported a bloodstream infection rate was 9,2 per 1,000 catheters/ day in developing countries that have similar difficulties in our country such as low compliance with hand hygiene and the nurse-to-patient staffing ratios in hospitals are low.

Of thirteen cases of bloodstream infections in our study, bacterial isolates from blood cultures revealed that $10(76.9 \%)$ were Staphylococcus epidermidis and 3 (23.07\%) were Staphylococcus aureus. The possible explanation for that finding been is attributed to poor compliance with skin cleaning with antiseptics around the catheter insertion site. So, Staphylococcus epidermidis is a Gram- negative bacterium normal flora that lives on the surface of the skin. This finding was consistent with [21] who conducted a study about Infection related processes during hemodialysis in general hospital and found that $77 \%$ of episodes of bloodstream infection were mainly due to Staphylococcus epidermidis.

As well, our finding is agreed with a recent similar study done by [22] who studied central venous catheter colonization and blood stream infection in a tertiary teaching hospital in India and revealed that negative staphylococci was the most common organism found causing central related blood stream infection .Moreover ,our finding was agreed with the results of previous studies done by [23-25] that have found that a dominance of Gram-negative bacteria that may be related to the colonization of the hands of healthcare providers in that hospitals. Similarly, our data are supported by similar study done by [26] who displayed domination of negative bacteria causing blood stream infection among children in ICU. On the hand, our finding is contradicted with a similar study done [27] who found gram positive co cci were the most common organisms colonizing CVCs followed by Gram negative Bacteria.

Regarding cultures sensitivity of microorganisms to antibiotics, our findings revealed $S$ aureus and $S$ epidermidis that isolated from nosocomial BSI cases were sensitive to Vancomycin, Amikacin and levofloxacin. This finding is partially in line with similar study done by [28] who reported that all resistant Staphylococcus isolated from CRLI were 100\% sensitive to vancomycin, teicoplanin, and linezolid . Moreover, our finding is congruent with the results of the study done by [29] that conducted a study about Catheter-related

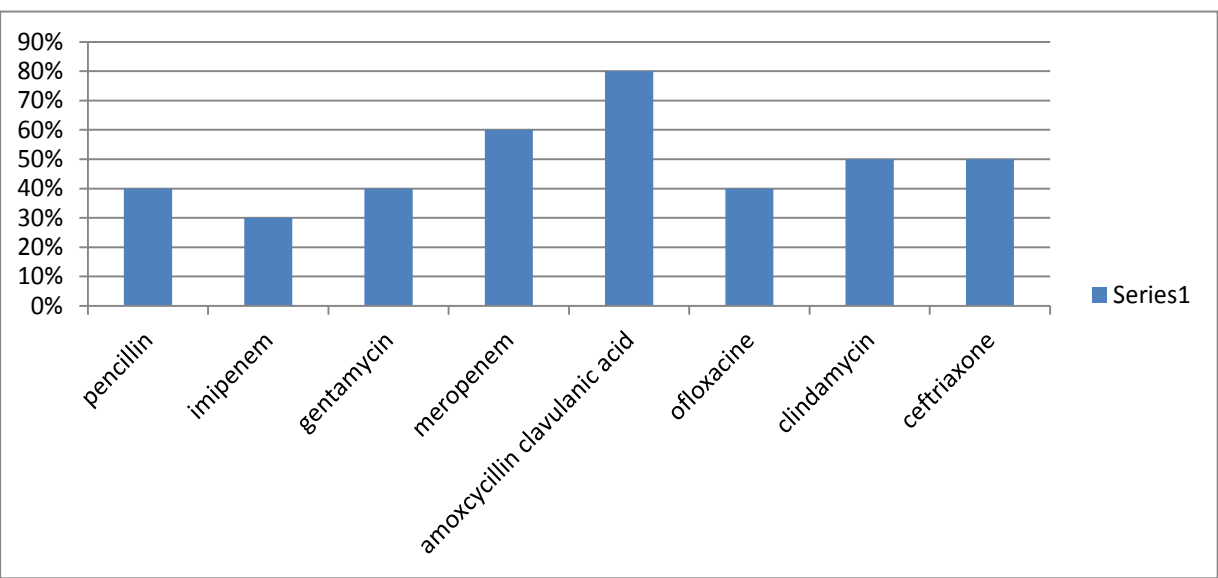

FIGURE 2. Antibiotic resistance of S. epidermidis isolates and S.aureus 
bloodstream infection in the intensive care unit and found all isolates were sensitive to Vancomycin and Teicoplanin. Concerning studying the antibiotic resistance of $S$. epidermidis isolates and S.aureus in the current study, it revealed that the majority of cases were resistent to Amoxycillin and Meropenem, on the other hand, half of cases were resistent to Olfloxacine, clidamycin and ceftriaxone.

Possible hazard factors for central line blood stream infection have been quoted in earlier studies, e.g., catheter type, time length of catheterization, site of insertion, duration of hospital stay, triple lumen catheter, and Duration of catheter in place. In our study, we observed an overall average of hospital stay was 10 days and all the cases stayed more ten have been associated with blood stream infection .This finding is supported by [23] who studied Epidemiology of nosocomial bloodstream infections in Estonia and reported that increased hospital stay duration have been related to increased BSI estimates.

Whether the number of lumens in the CVCs was linked with a greater incidence of infection is questionable. In our study, the nurses utilized triple lumen for all engaged patient . The possible explanation for selecting triple lumen was administration of parental fluids, medications, blood products and measuring CVP. Therefore, catheter was manipulated multiple times per day. Our finding is in agreement with [30,31] who observed in his analysis that the possibility to catch bloodstream infection was high when a patient had utilized numerous -lumen CVC in comparison to a single-lumen catheter.

Referring to the location of patients' insertion of central venous catheters, our study findings revealed that all cases were inserted in the ICU setting and nearly half of them were in emergency situations. Accordingly, the incidence of infection was higher. The possible explanation is optimal attention to aseptic technique during the time of insertion in emergency cases might not be Feasible and the catheters can be accessed multiple times per day for hemodynamic measurements or to obtain samples for laboratory analysis. Besides the dominance of nosocomial infections in ICU, our results are in line with [32] who reported that the occurrence of infection is often higher in ICU setting than in the less acute setting.
They interpreted the reasons as; central venous access might be needed for long periods of time; patients can be colonized with intensive care -acquired organisms; and the catheter can be handled many times daily for administering fluids, medications, and blood transfusion.

It has been validated that the insertion site of central venous catheter may be hazardous for the induction of blood stream infection [25]. Also, jugular venous catheters are liable to acquire infection than sub-clavian catheters. This may have relevance to mediators that promote colonization of the skin near to jugular vein such as coughing of sputum, difficulties in catheter fixation and frequent dressing [18].

As well, [33] studied a meta-analysis of central venous catheter-associated infection risk reduction in critically ill patients and revealed that the sub-clavian catheter site was combined with minor catheter-related infections compared with other sites. In our study, nearly more than half of cases (63\%) underwent jugular route. However, the incidence of blood stream infection was higher in patients with sub-clavian route. The possible explanation for this finding may be related to difficulty of technique of subclavian insertion that requires more expertise during insertion. As well, insertion of CVC catheter utilizing sub-clavian route took more than 20 minutes during insertion utilizing guide wires.

Technically, Central venous catheter insertion is often difficult and challenging in obese patients leading to obscured landmarks of the neck [34]. In addition, excessive sweating [35], dressing at the site of CVC insertion are frequently associated with problems and lead to higher risk of blood stream infection [17]. In our study, more than half of blood stream infection cases were overweight and obese. Our finding is contradicted with [36] who compared the CRBSI rate in obese and in non-obese and found no differences in CRBSI.

In our study, the CR-BSI rate in patients with premorbid disease such as diabetes mellitus was higher when compared to overall population. The possible explanation from the researcher's point of view, those diabetic patients may have low immunity and the CVC site of insertion is good media for entry of microorganisms. These findings are consistent with that of [37] who conducted a study about Clinical outcomes and 
economic variables in critically ill patients with bloodstream infections and found the rates of diabetes mellitus were greater among patients with BSI compared to patients without BSI.

\section{Conclusion}

The study concluded that out of 120 patients in the present study, 10\% developed bacterial blood stream infection 7 days after insertion of central venous catheters. and bacterial isolates from blood cultures revealed that three thirds of isolates were Staphylococcus epidermis .Moreover, , the incidence of central related blood stream infection cases was higher in patients with the subclavian route ,obese , diabetic and experienced more than seven days .Finally, our finding revealed that all bacterial isolates were sensitive to Vancomycin, Amikin, and Levofloxacin while most of bacterial isolates were resistant to Amoxycillin Clavulanic acid, Meropenem, Clindamycin and ceftriaxone, Penicillin and Ofloxacine and Imipenem. Our study was limited to generalize the results due to small sample size confined to one hospital. Therefore, our study recommended replication of the study on a larger probability sample from different geographical locations.

In summary, central venous catheters infection is still a major problem. Early detection and management are necessary to decrease the morbidity and mortality. This study showed the incidence, the risk factors and the bacteria isolated from patients defined to have such infections in a ministry of health hospital. Despite advances in management, this common clinical problem could still benefit from future breakthroughs.

\section{Limitations}

The main limitations of our study were that; conducted at a single center, on a small convenient sample of patients and may not be generalizable to other settings

\section{Acknowledgments}

We are grateful to all laboratory and nursing staff at Al Haram university hospital for their sincere cooperation.

\section{Funding}

No funds.

Availability of data and materials

Not applicable.

\section{Authors' contributions}

The authors designed, and revised tools for data collection. They helped in obtaining blood culture from patients. Later, they wrote the manuscript, analyzed and prepared the figures and illustrative tables. Later, they edited the manuscript to be ready for publication.

\section{Ethics approval and consent to participate}

The authors obtained the ethical approval from local ethical committee at faculty of nursing, Cairo University. As well, the written consent form was obtained from patients.

\section{Consent for publication}

All authors approve the manuscript submitted for publication.

\section{Competing interests}

The authors declare that they have no competing interests.

\section{Availability of supporting data}

The ethical approval and patients consent forms are present and signed. 


\section{REFERENCES}

Kelly LJ, Green A, Hainey K. Implementing a new teaching and learning strategy for CVAD care. Brit. J. Nurs. 24(8), S4-8, S9, S12 (2015).

Chopra V, O'Horo JC, Rogers MA, Maki DG, Safdar N. The risk of bloodstream infection associated with peripheral-ly inserted central catheters compared with central venous catheters in adults: a systematic review and metaanalysis. Infect. Control Hosp. Epidemiol. 34, 908-918 (2013).

Mlinar S, Rašković-Malnaršić R. Knowledge of nursing students about central venous catheters. Vojnosanitetski pregled 69, 333-339 (2012).

Tomlinson D, Mermel LA, Ethier MC, et al. Defining bloodstream infections related to central venous catheters in patients with cancer: a systematic re-view. Clin Infect Diseases. 53, 697-710 (2011).

Alkubati SA, Ahmed NT, Mohamed ONE, Fayed AM, Asfour HI. Health care workers' knowledge and practices regarding the prevention of central venous catheter-related infection. Am. J. Infect. Control. 43, 26-30 (2015).

O'Grady NP, Alexander M, Dellinger EP, et al. Healthcare Infection Control Practices Advisory Committee. Guidelines for the prevention of intravascular 2002 catheter-related infections. Centers for Disease Control and Prevention. MMWR Recomm. Resp. 51(RR10), 1-26 (2002).

Frasca D, Dahyot-Fizelier C, Mimoz O. Prevention of central venous catheterrelated infection in the intensive care unit. Crit. Care. 14(2), 212 (2010).

Snarski E. Current practices used for the prevention of central venous catheterassociated infection in hematopoietic stem cell transplantation recipients: a survey from the Infectious Diseases Working Party and Nurses' Group of EBMT. Transpl. Infect. Dis. 17(4), 558-565 (2015).

http://www.jsncare.uff.br/index.php/ jsncare/article/view/2725/0

Ullman AJ, Long DA, Rickard, CM. Prevention of cen-tral venous catheter infections: A survey of paediatric ICU nurses' knowledge and practice. Nurse Edu Today. 34, 202-207 (2014).

El-Kholy A, Tamer S, Gaber M, et al. Device-associated nosocomial infection rates in intensive care units at Cairo University hospitals: First step toward initiating surveillance programs in a resource-limited country, Egypt. Am. J. Infect. Control. 40, 216-220 (2012).

http://fac.ksu.edu.sa/sites/default/ files/Book-District_Laboratory_Practice_ in_Tropical_Countries_Part-2_Monica_ Cheesbrough.pdf

Lawshe $\mathrm{CH}$. A quantitative approach to content validity. Personnel Psychol. 28, 563-575 (1975).

Yaghmaie $\mathrm{F}$.Content validity and its estimation. J. Med. Edu. 3, 25-27 (2009).

Polit DF, Beck CT, Owen SV. Is the CVI an acceptable indicator of content validity? Appraisal and recommendations. Res Nurs Health. 30, 459-467 (2007).

Culshaw N, Glover G, Whiteley C, et al. Healthcare-associated bloodstream infections in critically ill patients: descriptive cross-sectional database study evaluating concordance with clinical site isolates. Ann. Intensive Care. 4, 34 (2014).

Trick WE, Miranda J, Evans AT, et al. Prospective cohort study of central venous catheters among internal medicine ward patients. Am. J. Infect. Control. 34, 636641 (2006).

Tacconelli E, Smith G, Hieke K, et al. Epidemiology, medical outcomes and costs of catheter-related bloodstream infections in intensive care units of four European countries: literature and registry-based estimates. J Hosp Infect. 72, 97-103 (2009).

Rosenthal VD, Maki DG, Mehta A, et al. The International Nosocomial Infection Control Consortium (INICC): Goals and objectives, description of surveillance methods, and operational activities. Am. J. Control. 36(9), 627-637 (2008).

Rosenthal VD, Maki DG, Salomao R, et al. International Nosocomial Infection Control Consortium. Device-associated nosocomial infections in 55 intensive care units of 8 developing countries. Ann Intern Med. 145, 582-591 (2006).

Hussain NF, Salama M, Yehia N, et al. Infection related processes during haemodialysis: A study in General Hospital Haemodialysis unit, 2010, Egypt.

Singh KM, Mallan D, Tripathi SS, Yadav RR, Avasthi S. A study of central Venous Catheter Colonizations and Bood stream Infections among patients admitted in the Intensive Care Unit of a Tertiary Care Teaching Hospital 2016.

Mitt P, Adamson V, Loivukene K, et al. Epidemiology of nosocomial bloodstream infections in Estonia. J. Hosp. Infect.71, 365-370 (2009).

Van der Kooi, Boer AS, Mannien J, et al. Incidence and risk factors of deviceassociated infections and associated mortality at the intensive care in the Dutch surveillance system. Intensive Care Med. 33(2), 271-278 (2007).

Lorente L, Henry C, Martin MM, et al. Central venous catheter related infection in a prospective and observational study of 2,595 catheters. Crit. Care. 9(6), R631635 (2005).

Abramczyk ML, Carvalho WB, Carvalho ES, et al. (Nosocomial infection in a pediatric intensive care unit in a developing country. Braz. J. Infect. Dis. 7(6), 375-380 (2003).

Kaur M , Cupta V, Gomhar S, Chander J, Sahoo T. Incidence ,risk factors ,microbiology of venous catheters associated blood stream infections - a prospective study from a tertiary care hospital . Indian J. Med. Microbiol. 33(2), 248-254 (2015).

Khanna V, Mukhopadhayay C, Vandana KE, Verma M, Dabke P. Evaluation of Central Venous Catheter Associated Blood Stream Infections: A Microbiological Observational Study. J. Pathogens. Article ID 936864, (2013).

Curtis RL. Catheter-related bloodstream infection in the intensive care unit. Intensive Care Society J. 10(2) (2009).

Moretti EW, Ofstead CL, Kristy RM, et al. Impact of central venous catheter type and methods on catheter-related colonization and bacteremia. J. Hosp. 
Infect. 61, 139-145 (2005).

Templeton A, Schlegel M, Fleisch F, et al. Multilumen central venous catheters increase risk for catheterrelated bloodstream infection: prospective surveillance study. Infect. 36, 322-327 (2008).

O'Grady NP, Alexander M, Burns LA, et al .Guidelines for the prevention of intravascular catheter-related infections. Clin. Infect. Dis. 52, e162-e193 (2011).

Parienti JJ, du Cheyron D, Timsit
JF, et al. Meta-analysis of subclavian insertion and non-tunneled central venous catheter-associated infection risk reduction in critically ill adults. Crit Care Med. 40(5), 1627-1634 (2012).

Graham AS, Ozment C, Tegtmeyer K, Lai S, Braner DA. Central venous catheterization. N. Engl. J. Med. 356, e21 (2007).

Lawrence VJ, Kopelman PG. Medical consequences of obesity. Clin. Dermatol. 22, 296-302 (2004).
Schalk E, Färber J, Fischer T, Heidel HF. Central Venous Catheter-Related Bloodstream Infections in Obese Hematologic Patients. Infect Control Hosp Epidemiol. 36(8) (2015).

Apostolopoulou E, Zikos D, Tselebis A, et al. Clinical outcomes and economic variables in critically ill patients with bloodstream infections. Health Sci. J. 8(4) (2014). 